\title{
Service - Oriented Architecture Model for Blood Type Analysis (Smart I (Eye) Advisory Rescue System) in Military Environment
}

\author{
Jurij F. Tasič ${ }^{1}$, Ana Madevska Bogdanova ${ }^{2}$, \\ Jugoslav Achkoski, ${ }^{3}$ and Aleksandar Glavinov ${ }^{4}$ \\ ${ }^{1}$ University of Ljubljana, Faculty of Electrical Engeneering, \\ Tržaška 25, SI-1000 Ljubljana, Slovenia \\ jurij.tasicafe.uni-lj.si \\ ${ }^{2}$ Faculty of Computer Science and Engeneering, \\ Rugjer Boshkovikj 16, P.O. Box 3931000 Skopje, Macedonia \\ ana.madevska. bogdanova@finki.ukim.mk \\ ${ }^{3,4}$ Military Academy "General Mihailo Apostolski", \\ Vasko Karangelevski bb, 1000 Skopje, Macedonia \\ jugoslav.ackoski@ugd.edu.mk, aglavinov@yahoo.com
}

\begin{abstract}
This paper proposes a model for telemedical Information System that can be used in a military environment. It is consisted of two modules: off-line Advisory Intelligent Module that obscures timely blood type recognition and an on-line module for distance interpretation of blood tests (determining the blood type) and fast delivering request to transport the injured in the nearest medical facility. The Smart I (eye) Advisory Rescue System (SIARS) will provide timely help to injured military persons or civilians, during or after military operations, and will provide correct blood serum analysis near the battlefield, as well as sending request for safest and fastest route to the nearest medical facility.
\end{abstract}

Keywords: SOA, Telemedicine, information system, military, modules and blood transfusion.

\section{Introduction}

Usually, during or right after the field combats, there is a need for blood infusion of the injured persons and it is done by using the neutral, type zero [12]. The idea is to find the way for fast blood serum analysis, and to provide the appropriate blood transfusion even in the local hospitals where no specialists are available, and/or during the transportation to the nearest (safest) medical facility. This can be done with the system of gel-cards [12]. The technician fills the gel-cards with the patient blood sample and obtains its image. The technician is a military medical person in the combat unit that executes military tasks. Dependent on the military doctrine, this person can be a part of a company or a section in a battalion. 
The interpretation of the gel-cards, according the traditional method for determining the blood type, is done by the specialist in the laboratory in a medical facility. The SIARS will broaden the usage of the Geloscope [12] by introducing additional information to the gel-card digital image of the injured (smart image). The specialist will read the blood type of the wounded person by the information from the smart image and deliver the needed information back to the technician. This scenario is possible only when on-line connection to the specialist exist. In the absence of this kind of connection, the Advisory Intelligent Module - AIM will take place. The SIARS will integrate both scenarios, in order to deliver exact blood type analysis in off-line and on-line mode.

\section{Related Work}

There are a large number of referred research papers from this area. Analyzing the relevant literature, we have drawn the conclusion that in many technologically developed countries the implementation of SOA in the telemedicine IS in the military domain is driven by the unique reason - to protect and save military person/civilian lives. The society benefits from the technology development by government founded research projects. The "Extending Service Oriented Architectures to the Deployed Land Environment" [1] shows that the interest about service-oriented architecture guides to extended implementation of SOA in information and communication systems, that are part of military and civil domain. Since it is simply implementing Service Oriented Architecture (SOA) [2], [3], [4], [5] base functionality into unchangeable organization infrastructure at the same time there are numerous factors that are more complicated implementing SOA in design of systems in military domain. The implementation of service-oriented architecture in systems for deployed land forces in missions which execute military operations or other kind of missions as a mission for establishing peace or keeping peace lead by NATO, EU or UN, represents a big challenge for its effective implementation in military domain.

In [6], information systems for command and control that are exploited in headquarters in an operational level use service oriented architecture in order to increase capability in exchanging information in military environment. SOA approach allows flexibility in increasing capability through integration and systems interoperability based on using Commercial-Off-The Shelf (COTS) technology and standards. A solution by Thales (UK), based on SOA is presented in the paper. It covers architecture modeling, SOA Governance and summary of a multinational demonstration activity used to implement prototype services. It concludes that SOA solution can be used for increasing the capability in military environment.

Also, as a further work, Thales proposes developing SOA solution of information systems of this kind, in all areas in military environment. The understanding business process domain will be tied to opportunities for the development of services focused on delivering improvements in capability to the customers. In addition, the solution is planned to be used for integration and interoperability with other information systems in military environment. 